\title{
Archéopages
}

Archéopages

Archéologie et société

Hors-série 3 | 2012

Nouveaux champs de la recherche archéologique

\section{La mixité sociale au travers des études céramologiques de corpus médiévaux et modernes d'île-de-France}

Fabienne Ravoire

\section{CpenEdition}

Journals

Édition électronique

URL : https://journals.openedition.org/archeopages/728

DOI : 10.4000/archeopages.728

ISSN : 2269-9872

Éditeur

INRAP - Institut national de recherches archéologiques préventives

Édition imprimée

Date de publication : 1 janvier 2012

Pagination : 193-199

ISSN : 1622-8545

\section{Référence électronique}

Fabienne Ravoire, "La mixité sociale au travers des études céramologiques de corpus médiévaux et modernes d'Île-de-France », Archéopages [En ligne], Hors-série 3 | 2012, mis en ligne le 01 janvier 2012, consulté le 26 février 2023. URL : http://journals.openedition.org/archeopages/728 ; DOI : https:// doi.org/10.4000/archeopages.728 
types de production, pour les utilisations

culinaires par exemple. Dans le cadre très précis de l'archéologie postérieure au Moyen Âge, la notion de mobilier remanié ou résiduel semble donc plus difficile à percevoir et à déterminer. Travailler sur la période moderne doit être forcément associé aux études des archives écrites. Pour illustrer cette portion de mobilier domestique, les inventaires après décès pourraient être d'un certain secours. Les sources lyonnaises sont quasi muettes et les mentions de céramiques sont toujours anecdotiques. Contrairement aux vaisselles de verre ou d'étain, les poteries ont une simple valeur d'usage et qui écarte toute revente, d'où peut-être leur absence dans ces textes, ce qui n'est pas forcément le cas cependant dans toutes les régions. À Lyon, les données archéologiques sont presque les seules disponibles pour aborder cette notion de part d'héritage dont témoignent ces vaisselles familiales entre les XVI ${ }^{\mathrm{e}}$ et XIX ${ }^{\mathrm{e}}$ siècles.

Alexandre-Bidon D., 2005 : Une archéologie du goût. Céramique et consommation (Moyen Âge, Temps Modernes), Paris, Picard.

Arlaud C., Lurol J.-M., Savay-Guerraz S., Vérot-Bourrély A., Auger-Rahatsöz M., Ayala G., Bernardi P., Bertrand E., Bonnet C., Cécillon C., Forest V., Horry A., Le Nézet M., Plantevin C., Rolland M., Vaireaux F., Vicard T., 2000 : « Lyon, les dessous de la Presqu'île. Bourse-République-CélestinsTerreaux. Sites Lyon Parc Auto ", Documents d'Archéologie en Rhône-Alpes, $\mathrm{n}^{\circ}$ 20, Lyon.

AYAlA G. (DIR.), à paraître : La fouille du Parc Saint-Georges à Lyon. Formation et évolution d'un espace fluvial en bord de Saône, DAF.

COLleCtif, 1990 : À la fortune du pot. La cuisine et la table à Lyon et à Vienne ( $X^{e}$-XIX $X^{e}$ s.), Catalogue de l'exposition Lyon-Vienne-Mâcon, 1990-1991.

Faure-Boucharlat E., Vicard T., Maccari-Poisson B., SavayGuerraz S., 1996 : Pots et potiers en Rhône-Alpes. Époque Médiévale. Époque Moderne, DARA, 12, Lyon, Service régional de l'archéologie, DRAC Rhône-Alpes, $315 \mathrm{p}$

Faure-Boucharlat E., 2004: « Archéologie urbaine et époque moderne : quelques réflexions à partir d'expériences lyonnaises », Les nouvelles de l'archéologie, 96, p. 17-31.

Horry A., 2001 : « La faïence à Lyon du XIV ${ }^{\mathrm{e}}$ au XVI ${ }^{\mathrm{e}} \mathrm{s}$. : l'apport des fouilles récentes ", Archéologie du Midi Médiéval, t. 19, p. 137-179.

HORRY A., 2010 : «Terra incognita? Céramiques et archéologie des Temps Modernes : premier bilan et réflexions à partir de l'exemple de Lyon », in JeAn CHAPELOT (DIR.), Trente ans d'archéologie médiévale en France. Un bilan pour un avenir, Actes du IX ${ }^{\mathrm{e}}$ congrès international de la Société d'archéologie médiévale, Caen, Publications du Crahm.

HORRY A., 2011 : «Terres lyonnaises. Les objets de terre cuite au XVIII ${ }^{\mathrm{e}}$ s. », Archéologia, n ${ }^{\circ} 485$, p. 36-50.

Horry A., 2012 : Poteries de Lyon. 1500-185o. Morceaux choisis du quotidien à Saint-Georges, Lyon, éditions Lyonnaises d'Art et d'Histoire, Inrap.

Journot F., Bellan G. (DIR.), 2011 : Archéologie de la France moderne et contemporaine, Paris, La Découverte, 2011.

RAVOIRE F., 2007 : « Mobilier céramique des XVII ${ }^{\mathrm{e}}$ et XVIII ${ }^{\mathrm{e}}$., approche sociale: l'apport des fouilles archéologiques d'Île-de-France », in Collectif, Actes du Colloque Medieval Europe in Paris, Paris, 3-8 septembre 2007. Cf. http://medieval-europe-paris-2007.univ-paris1.fr
La mixité sociale au travers des études céramologiques de corpus médiévaux et modernes d'île-de-France

Fabienne Ravoire
Inrap

L a céramique constitue, avec les restes de faune, le mobilier le plus systématiquement retrouvé dans les contextes archéologiques d'habitats médiévaux et modernes. La confrontation des assemblages observés dans des sites de nature variée constitue un moyen pour comprendre la diffusion des productions sur un espace donné. L'Île-de-France, région pour laquelle les fouilles, en particulier préventives, de sites de ces périodes sont engagées depuis une quarantaine d'années, se trouve donc très bien documentée avec notamment de très grosses opérations comme à Saint-Denis (Seine-Saint-Denis), autour de la basilique, à Paris, d'abord sur le parvis de la cathédrale Notre-Dame, puis au Grand Louvre : trois opérations couvrant l'ancien château du Louvre jusqu'à l'emplacement du palais des Tuileries, soit une surface de près de 10 hectares. On compte encore, des années 1990 à 2000, quelques belles opérations dans la capitale (la cour du Collège de France, les thermes de Cluny, le boulevard Saint-Michel, sur la rive gauche, le boulevard Sébastopol et plusieurs parcelles bâties dans le quartier des Halles et du Marais, sur la rive droite). Depuis, les opérations se portant moins dans Paris que dans de nombreuses villes de la région, comme Meaux et Lagny (Seine-et-Marne) à l'est, Étampes (Essonne) au sud, Argenteuil à l'ouest (Val-d'Oise). Des interventions dans des milieux particulièrement prestigieux comme le château des Tuileries (appartements des proches de la reine), le château de Versailles (cour des Grands Communs), le château de Vincennes ou, encore en cours de fouille, le camp d'entrainement de Louis XIV à Saint-Germain, apportent leurs lots de mobiliers particulièrement représentatifs des modes de consommation dans ces contextes d'élite. Ceux-ci peuvent être comparés à des milieux plus simples comme une auberge à Étampes, une ferme à Varennes, des maisons villageoises à Roissy, Ivry, Tremblay, des maisons urbaines et péri-urbaines à Paris etc.

De ce fait, le nombre des céramiques médiévales et modernes dont nous disposons en Île-de-France pour étayer des études se situe parmi les plus importants de France. La recherche céramologique qui s'est développée depuis les années 1990 porte, comme à peu près partout en France, sur l'identification, la caractérisation et la datation des productions céramiques, mais également sur des aspects plus culturels de ce mobilier (comme la question de la fonction). Des classements plus ou moins efficients étant opérés sur la base de traces d'usages et aussi d'une plus ou moins grande proximité avec des céramiques identifiées dans les répertoires de l'ethnographie française (voir, 
par exemple pour l'Île-de-France, Ravoire 2006a et 2006b). En ce qui concerne la fonction des récipients, et pas seulement les céramiques, la question a été posée dans un colloque sur la table à la fin du Moyen Âge en 2004. (Ravoire, Dietrich, 2009). L'usage de la céramique médiévale a ensuite fait l'objet d'une étude tout à fait essentielle (Alexandre-Bidon, 2005).

\section{Un axe de recherche encore peu développé :}

l'approche sociale. L'interprétation sociale des objets est un axe de recherche peu développé en France dans le domaine de l'archéologie médiévale et moderne. Dans d'autres domaines archéologiques, certains chercheurs se sont démarqués en proposant des modèles sociétaux résolument tournés vers des approches d'anthropologie sociale (Baray, 2003 ; Baray et al., 2007). Chez les historiens, cette orientation est également développée par les chercheurs qui se placent dans le champ de l'histoire culturelle, à la suite de l'historien Fernand Braudel, qui, dans son ouvrage Les structures du quotidien : le possible et l'impossible, a permis un autre questionnement sur la société que celui directement lié aux structures politiques et économiques. L'historiographie, essentiellement moderne, a ainsi pris en compte la culture du quotidien dans son champ d'investigation, en particulier par le développement de l'étude des objets liés à des groupes sociaux différents, dans une perspective d'histoire sociale ou anthropologique (Roche, 1981 ; Garnot, 1995). En France, les chercheurs se sont surtout intéressés aux objets dans le cadre d'une histoire de la vie privée, alors que les chercheurs anglo-saxons se sont orientés sur l'anthropologie de la consommation. Cette approche culturelle est développée par des historiens modernistes qui se placent dans la mouvance développée par D. Roche (Bourquin, Hamon, 2011, p. 11). Les inventaires après décès constituant la principale source de ces recherches historiques. Les études menées par exemple sur les sources parisiennes montrent qu'il existe une hiérarchie des objets en fonction de leur qualité (Pardailhé-Galabrun, 1989; Roche, 1981). L'étude des biens culturels au sein d'une même catégorie socio-professionnelle, comme a pu le montrer Laurent Bouquin sur les officiers du parlement et du Châtelet de Paris dans la première moitié $\mathrm{du} \mathrm{XVI}^{\mathrm{e}}$ siècle, est particulièrement significative : la qualité des objets et des matériaux constituent les critères les plus évidents de la distinction sociale (Bourquin, 1989, p. 471). De nombreux objets (vaisselle métallique, bijoux, tissu...), par leur coût, leur rareté, ont pu ainsi apparaître comme des marqueurs statutaires, mais, selon le milieu concerné, ils n'avaient pas forcement le même impact. Une partie plus ou moins importante du mobilier, et notamment des ustensiles, n'entrait pas en ligne de compte car elle était considérée comme ayant trop peu de valeurs par les héritiers ; néanmoins, la vaisselle d'étain faisait partie des ustensiles qui étaient systématiquement dénombrés, quel que soit le statut social de ses propriétaires (Bourquin, 1989, p. 466, 471). Ce constat est également celui de $\mathrm{S}$. Pendery sur les inventaires après décès des populations urbaines des $\mathrm{XVII}^{\mathrm{e}}$ et XVIII ${ }^{\mathrm{e}}$ siècles du Massachusetts (Pendery, 2007).

Le mobilier céramique dans les sources écrites. Le mobilier céramique est rarement signalé dans les inventaires avant le XVIII ${ }^{\mathrm{e}}$ siècle, sauf dans quelques régions comme en Bourgogne à la fin du Moyen Âge. La poterie de terre est un mobilier omniprésent dans l'équipement domestique et, mis à part des produits très spécifiques, son coût est si peu élevé qu'il n'est pas prisé par les notaires (sauf évidemment dans le cas des potiers de terre). Dans les inventaires de marchands potiers, il existe bien une valeur différentielle de la céramique selon la catégorie de céramique. Par exemple, à Paris, au $\mathrm{XVI}^{\mathrm{e}}$ siècle, les potiers parisiens désignent des pots plombés (leur production) et les pots de Beauvaisis (Ravoire, 2011, p. 118). Dans les inventaires parisiens du XVII ${ }^{\mathrm{e}}$ siècle apparaissent les faïences et une autre catégorie de céramique, la poterie de Flandres (grès rhénans). Un inventaire du milieu du XVIII ${ }^{\mathrm{e}}$ siècle d'un marchand de poterie de Mantes (Yvelines) indique clairement qu'au sommet de la hiérarchie des céramiques qu'il vend se trouve la faïence décorée, puis la faïence blanche, vient ensuite la céramique avec glaçure («potterie plombée») et enfin celle sans glaçure («potterie commune ») et le grès (Waro-Desjardins, 1996, p. 161). À Dijon, dans les inventaires après décès de potiers des $\mathrm{XIV}^{\mathrm{e}}$ et $\mathrm{XV}^{\mathrm{e}}$ siècles, les «pots» sont qualifiés de «noirs », de « rouges» et de «verts». Les premiers viennent de Sevrey ( «Sevrer») en Saône-et-Loire, les seconds des officines locales de Villers-les-Pots («Villers Lespotx») et de SaintSeine l'abbaye ( «Saint Seigne») et les derniers du Jura d'Etrepigney («Estrepignier»). Un inventaire de 1410 d'une potière précise que les pots noirs représentent les deux tiers du stock (Piponnier, 1987). L'étude des contextes archéologiques dijonnais contemporains n'offre pas tout à fait la même image. Certes, les pots noirs sont bien présents au $\mathrm{XV}^{\mathrm{e}}$ siècle (deux fois plus d'ailleurs qu'au XIV ${ }^{\mathrm{e}}$ siècle), mais les pots rouges sont les plus nombreux à cette époque. En revanche, ils ne dominent, dans les contextes archéologiques, que dans la première moitié du XVI ${ }^{\mathrm{e}}$ siècle (Ravoire, 2011). Au XVIII ${ }^{\mathrm{e}}$ siècle, les inventaires sont plus nombreux qu'auparavant, et la faïence, qui a acquis, depuis le $\mathrm{XVII}^{\mathrm{e}}$ siècle un statut supérieur à celui de la poterie de terre, est régulièrement mentionnée. C'est justement à cette époque que la vaisselle en faïence est massivement fabriquée dans la plupart des régions de France avec, d'un côté, une production ordinaire et, de l'autre, une production haut de gamme (cette dernière ne se trouvant pas dans toutes les manufactures). Ces deux qualités sont clairement identifiées dans les ensembles archéologiques.

En dehors, de ces mentions dans les inventaires ou dans de rares comptabilités d'approvisionnement de bouche, la céramique est rarement mentionnée, où alors de manière si peu précise qu'il est souvent difficile d'en tirer des informations d'ordre qualitatif. Quand des mentions figurent, elles sont rarement
2 C'est la thématique d'une recherche sur laquelle nous travaillons dans le cadre de la préparation d'une HDR. 
en concordance avec les sources archéologiques. Par exemple, dans un inventaire après décès de 1742 d'un fermier de la ferme du Colombier, à Varennes, seuls quelques pichets en grès sont mentionnés dans la laiterie. Or nous avons retrouvés sur place la quasi-totalité du vaisselier de l'époque, qui était fort abondant et destiné tant à la laiterie qu'à la vaisselle d'usage parmi laquelle figurait de nombreuses faïences (Ravoire, 2012). Dans un autre inventaire, qui date du début du XVIII ${ }^{\mathrm{e}}$ siècle, les mentions de vaisselle sont au contraire nettement plus importantes quantitativement que le mobilier réellement trouvé dans la fouille du château de Roissy-en-France (Ravoire, à paraître). Une différence majeure explique aussi cela : dans le premier cas, la vaisselle est celle d'un fermier, certes pourvu d'un peu de biens, mais elle n'a pas retenu l'attention du notaire $d u$ fait de sa faible valeur marchande, tandis que, dans le second cas, il était notable d'exposer l'importance des services de porcelaines chinoises et de faïences rouennaises du comte de Caraman, propriétaire du château ! La confrontation avec les sources textuelles, quand elle est possible (comptabilités, inventaires après décès..), permet donc des éclairages intéressants, mais qui sont à nuancer dans le cadre d'une appréciation du niveau de vie des utilisateurs...

Quel statut pour l'objet céramique? Les objets archéologiques et parmi eux, la céramique, peuvent-ils nous renseigner sur le statut social des utilisateurs ? Comment estimer la valeur de la céramique comme bien de consommation à partir des contextes archéologiques? La méconnaissance que l'on peut avoir des véritables utilisateurs des céramiques qui ont été rejetées, quand bien même le statut de l'habitat est identifié, constitue le principal écueil à une interprétation sociale du mobilier céramique (Husi, 2009, p. 278), ce qui n'est pas le cas des inventaires après décès. Pourtant, cette approche sociologique, qui consiste à se poser la question du sens à donner aux objets archéologiques, à les comprendre dans l'idée d'une matérialisation de la culture, s'avère une piste de recherche à ne pas négliger. Cette recherche ne peut cependant se faire qu'avec une méthodologie ad hoc, sur un corpus abondant et diversifié (Ravoire, 2007b)2. Des approches ont été réalisées sur le mobilier d'époque moderne en Île-de-France, notamment sur des catégories fonctionnelles de récipients relativement bien identifiés, la vaisselle de table et

3 Dans le cas de la céramique, il s'agit de la transposition en terre cuite de modèles en métal plus onéreux. la vaisselle de toilette (Ravoire, 2002, 2007b, 2008). En Angleterre (Courtney, 2010), en Belgique (Verhaeghe, 1996 ; Burnouf et al., 2009), ce type de questionnement par l'utilisation de modèles et de concepts d'anthropologie sociale est déjà d'actualité depuis plusieurs années. Pour autant, à notre connaissance, aucune grande synthèse mettant en évidence les mécanismes de distribution du mobilier céramique dans cette perspective et sur la longue durée n'a encore été réalisée dans ces pays.

Il nous semble d'abord nécessaire d'aborder des aspects qui tiennent aux processus mêmes d'acquisition des objets : qui les fabrique et pour qui ? Quels sont les liens entre des objets de fonctions similaires et de matières différentes ? Le phénomène des «skeuomorphes» ${ }^{\mathbf{3}}$, que F. Verhaeghe a mis en évidence sur les corpus de céramiques médiévales belges (Verhaeghe, 1996), manifeste qu'il n'y a pas de limite nette entre, d'une part, l'idée que l'on pouvait se faire (au Moyen Âge et dans les siècles suivants), d'un type de récipient associé un usage particulier (si ce n'est le matériau dans lequel il était réalisé) et le coût de sa mise en œuvre. Ainsi, par exemple, rien ne distingue, dans la forme, une assiette à aile du $\mathrm{XVI}^{\mathrm{e}}$ siècle, qu'elle soit en bois tourné, en céramique, en étain, ou en argent. A-t-il existé comme le suggère F. Verhaeghe, une concurrence inter-artisanale? La réponse ne peut être que nuancée. On remarque des décalages, par exemple pour la vaisselle d'usage, entre la circulation de modèles en métal et celui de leur équivalent fonctionnel en terre cuite : de fait, un monde sépare cette vaisselle d'usage de la vaisselle d'apparat, par essence non utilitaire, à laquelle de rares catégories de céramiques ont pu ponctuellement appartenir (ainsi, les faïences fines dites de Saint-Porchaire ou celles de Bernard Palissy (Crepin-Leblond, 1998). Prenons l'exemple de l'assiette telle qu'elle apparaît en France au $\mathrm{XVI}^{\mathrm{e}}$ siècle, dans la plupart des productions locales. Cette forme en bois semble également apparaître au XVI ${ }^{\mathrm{e}}$ siècle (Dietrich, 2009, p. 159), mais elle existe aussi en métal dans la vaisselle d'apparat du XIV ${ }^{\mathrm{e}}$ siècle, désignée dans un inventaire de 1364 comme « écuelle à large bord » (Muel, 2011, p. 110). Cette forme est cependant aussi répandue dans la vaisselle utilitaire au XVI ${ }^{\mathrm{e}}$ siècle : en argent chez les grands seigneurs qui possédaient des services, numériquement importants, d'assiettes souvent à leurs armes (Bimbenet-Privat, 2009, p. 153-154) et chez les riches bourgeois (Bimbenet-Privat 2011), et en étain chez les autres.

Les consommateurs agissaient donc en fonction de leur moyen et de leurs besoins. Seuls, les plus fortunés, quel que soient leur statut, bourgeois ou aristocratique, avaient pour autre mobile de consommation le paraître, la représentation, la nécessité d'avoir ce que, à statut égal, les autres ont, mais aussi, pour une frange plus étroite, d'avoir ce que les autres n'ont pas. C'est la raison pour laquelle les critères de rareté, de nouveauté du matériau, en relation souvent avec des savoir-faire techniques élaborés, ont abouti à la fabrication de produits «haut de gamme», recherchés par des clientèles fortunées destinées à se distinguer du reste de la société. V. Abel, au sujet des céramiques de Marseille, ne dit pas autre chose : « rares sont celles qui arrivent à résister sans une fonction spécifique que ne peuvent remplir les poteries locales [...]. Ce serait [...] l'usage qu'on réservait à un vase qui déterminait le choix à l'achat [et non pas nécessairement des prix plus bas]. Le besoin d'une vaisselle moins ordinaire que les terres vernissées locales utilisées quotidiennement poussait à "acheter italien" ; la nécessité de cuisiner obligeait à acquérir des poteries de diverses origines, les fabriques locales ne produisant plus de céramiques culinaires »(Abel, 1987, p. 165). 
Le regard sur les mécanismes d'acquisition des biens matériels porté par les historiens est très intéressant. Par exemple, le fait qu'à la période moderne, «la consommation des élites se traduit par un désir d'accumulation », plus que par la manifestation d'un luxe outrageux (Bourquin, Hamon, 2011, p. 13), fait résonnance à ce que l'on peut observer dans nos contextes archéologiques. «Certaines formes de consommation apparaissent donc comme de véritables marqueurs sociaux qui fédèrent les élites, quelles qu'elles soient, autour de pratiques et de valeurs communes : la quête du confort, le loisir, la détente, l'attention portée à la mode... Elles nous permettent de mieux appréhender les processus de distinction sociale qui s'affirment dans les villes dès la Renaissance, et sur lesquelles les sources se multiplient à partir du début du XVIII ${ }^{\mathrm{e}}$ siècle» (Bourquin, Hamon, 2011, p. 12-13).

\section{La recherche de marqueurs statutaires au sein} du vaisselier céramique. L'archéologie est à même, à partir de ses propres données, de proposer des estimateurs de la richesse sociale, à la condition que ces « indicateurs » soient perçus au sein d'une entité géographique, politique, clairement définie. La valeur de certains biens, il en va pour la céramique comme d'autres objets, n'est pas nécessairement la même dans toutes les régions de France à la même période. C'est la recherche des variables propres au mode de consommation de chaque type d'objet qui est déterminante.

Pour donner un sens à l'objet, il faut déterminer les similarités et les différences (Hodder, 1987). C'est la raison pour laquelle, il est nécessaire de comparer des lots contemporains, de milieux semblables d'abord, puis différents dans un second temps. C'est donc avant tout en multipliant des études de site de nature semblable et en les comparant que l'on peut obtenir l'image la moins déformée possible de cette réalité matérielle. En effet, le problème tient aux contextes des découvertes, des dépotoirs dans la majorité des cas, qui ne reflètent pas de manière aussi évidente le mobilier en usage dans la haute aristocratie : il peut s'agir de la vaisselle de la garnison comme au château de Vincennes (dépotoir de la tour des Salves), celui des prisonniers du château (dépotoirs des XVI ${ }^{\mathrm{e}}$ et XVII ${ }^{\mathrm{e}}$ siècles de la cour du donjon), celle des domestiques qui occupent les communs de châteaux comme ceux du Louvre. Par ailleurs, le statut même de ces châteaux différents et l'on ne peut comparer qu'avec prudence les corpus des châteaux de Versailles ou de des Tuileries avec ceux du château de Roissy-en-France pour citer des exemples extrêmes. Ensuite, c'est en comparant les entités de nature sociale différenciée que l'on aura une preuve tangible de la validité de ces recherches à partir du mobilier archéologique comme estimateur de richesse.

Comment, dans le cadre de la production artisanale de la poterie de terre, telle qu'elle pouvait être pratiquée au cours du Moyen Âge et sous l'Ancien Régime, avec ses réglementations, son organisation, son système de production et de vente, la recherche de produit «survalorisé » a-t-elle pu exister par rapport à la production de masse ? Car c'est bien-là le cœur de notre problème : savoir si, parmi toutes les céramiques qui ont été fabriquées durant toutes ces décennies, dans les innombrables officines régionales qui ont essaimé dans le territoire, des produits se sont distingués, apportant une plus-value à la poterie de terre d'usage courant. L'identification de ces céramiques, que d'aucuns qualifieraient de «luxueuses » parmi l'immensité des productions identifiées sur les sites archéologiques, constitue bien une des recherches prioritaire des céramologues. Quel crédit accorder à la céramique avec glaçure et avec peinture et polissage carolingienne? À la céramique à décor plastique rapporté et glaçure du Moyen Âge classique (dite très décorée) ? Au grès au bas Moyen Âge ? À la porcelaine et à la faïence à la Renaissance et à l'âge classique?

En ce qui concerne le domaine francilien, certaines catégories de céramiques ont été longtemps considérées comme des marqueurs statutaires. Pour la période carolingienne, par exemple, l'apparition, au $\mathrm{x}^{\mathrm{e}}$ siècle, de la céramique avec glaçure a longtemps été «le marqueur social» de la période parce que rare et donc considérée comme une céramique luxueuse (Lefèvre, Mahé, 2004, p. 137). Pourtant, que sait-on vraiment de la distribution de cette vaisselle, puisque l'essentiel des sites de cette période qui ont été fouillés sont des habitats ruraux, en dehors du bourg monastique de Saint-Denis. Force est de constater que ces céramiques, si elles sont de belle facture pour les pichets, sont par ailleurs assez ordinaires pour les bols ou les poêlons. En revanche, leur présence apporte une connotation culturelle forte, propre à l'Europe du Nord-Ouest, en relation avec des manières de table ou plutôt de consommer le vin (Husi, 2006, p. 178-179). Pour les deux siècles qui lui succèdent, c'est toujours cette céramique «hautement décorée et glaçurée » qui constitue le principal marqueur statutaire. Sa présence a été longtemps considérée comme attachée à une clientèle aisée, plutôt urbaine ou aristocratique. Là aussi, les contextes urbains sont extrêmement rares. La vaisselle glaçurée est bien présente dans certains dépotoirs du parvis de Notre-Dame, datés la deuxième moitié du XII ${ }^{\mathrm{e}}$ siècle (Nicourt, 1986, p. 168, p. 184-185, p. 193-194, p. 198). Dans les contextes ruraux, cette vaisselle semble encore bien rare. Par contre, on retrouve des pichets (principalement) du XII ${ }^{\mathrm{e}}$ siècle dans des contextes castraux, comme le château de la Madeleine à Chevreuse (Yvelines), Blandy-les-Tours (Seineet-Marne), Roissy-en-France (Val d'Oise). Cette vaisselle est aussi retrouvée dans l'atelier rural de Fosses (Val d'Oise) à cette époque (Guadagnin, 2007). Entre le milieu du XIII ${ }^{\mathrm{e}}$ et le milieu du $\mathrm{XIV}^{\mathrm{e}}$ siècle, la vaisselle avec glaçure n'est pas loin de recueillir 50\% du vaisselier urbain et, dans certains contextes aristocratiques et religieux, elle atteint jusqu'à 80\% des effectifs (Ravoire, 2000, p. 449 ; Durey-Blary, 1993, p. 137 ; Guadagnin, 2007). Elle est également attestée en milieu rural, faiblement 
avant le $\mathrm{XIV}^{\mathrm{e}}$ siècle et se généralise à partir de cette période comme le montre le site d'habitat de Tremblay-en-France «Le rue des Fossés » (Lafarge, Lefèvre, 2009, p. 432 et p. 435). Aussi à partir du XIII ${ }^{\mathrm{e}}$ siècle, la céramique glaçurée dans notre région ne constitue plus un marqueur statutaire, sauf pour des produits dont on peut penser que la qualité et la sophistication du décor a pu engendrer un surcoût notable (voir les exemples de Fosses ou de Saint-Denis : Guadagnin, 2007, p. 140-141 ; Nicourt, 1986, p. 113-117).

Le grès du Beauvaisis, produit céramique nouveau au XIV $\mathrm{X}^{\mathrm{e}}$ siècle, car moins poreux que la céramique ordinaire, semble avoir été d'abord perçu comme une curiosité. Les sources archéologiques tant en production qu'en contextes de consommation montrent que sa fabrication remonte à la première moitié du $\mathrm{XIV}^{\mathrm{e}}$ siècle. Pourtant, son caractère exceptionnel est encore perçu à la fin de ce siècle. Il figure en effet dans l'inventaire daté de 1399 du roi Charles VI : « un godet de terre de Beauvais garny d'argent» (Chami, 1963, p. 90). Il est possible, évidemment, que cet objet ait été déposé dans le trésor beaucoup plus tôt. Mais, dans un autre compte royal de 1416, il est également mentionné « plusieurs godez de Beauvez et autres vaisselles à boire » (Chami, 1963, p. 90). En Bourgogne, c'est à l'occasion de la venue en 1389, du duc de Bourgogne au château de Villaines-en-Duesmois, que sont achetés des «Godèz de Beauvais» (Piponnier, 1987, p. 241, note 42). Ce mobilier céramique peut donc être considéré comme relevant de la vaisselle d'usage utilisée par l'aristocratie au XIV et au début du $\mathrm{XV}^{\mathrm{e}}$ siècle. Que nous apprennent les contextes archéologiques contemporains? À Paris, les godets en grès ont été identifiés dans quelques dépotoirs urbains des fouilles de la cour Napoléon (Grand Louvre), mais on ne connaît pas vraiment le statut des occupants du secteur. Ces structures étaient associées à des habitats situés aux abords occidentaux du château du Louvre. Ces grès ont alors été datés de la fin du XIV ${ }^{\mathrm{e}}$ siècle puisque l'on s'appuyait sur les mentions écrites de cette période (Ravoire, Monnet 1992). Plus tard, la destruction du manoir seigneurial, vers 1355, ainsi que les remblais de systèmes défensifs de la ville, mis en place sensiblement à la même période, ont livré ce type de mobilier qui fut dès lors daté du second tiers du XIV ${ }^{\mathrm{e}}$ siècle (Ravoire, 1998a et 1998b). On ne peut pas vraiment parler de généralisation des grès du Beauvaisis au XIV ${ }^{\mathrm{e}}$ siècle. En revanche, à partir $\mathrm{du} \mathrm{Xv}^{\mathrm{e}}$ siècle, cette vaisselle, vendue par les marchands potiers parisiens, est de ce fait rapidement diffusée dans l'équipement domestique (Chami, 1963 ; Ravoire, 2011). En revanche, le statut du grès du Beauvaisis à Dijon est tout autre : en effet, il semble qu'il faille attendre le $\mathrm{XVI}^{\mathrm{e}}$ siècle pour que cette vaisselle, soit attestée dans les contextes archéologiques dijonnais (Ravoire, 2011). Les sources testamentaires étudiées par Françoise Piponnier pour les $\mathrm{XIV}^{\mathrm{e}}$ et $\mathrm{XV}^{\mathrm{e}}$ siècles ne font pas mention de ce type de céramique (Piponnier, 1987) et nous-même n'en avons pas trouvé dans les occupations archéologiques de cette même période (mais elles sont certes beaucoup plus rares qu'à Paris !) (Ravoire, 2011). Les études céramologiques futures dans la région permettront sans doute de mieux apprécier la place de ce mobilier céramique comme marqueur statutaire dans la «culture matérielle» médiévale bourguignonne.

À la période moderne (Journot et al., 2011), plus qu'au Moyen Âge, l'époque est propice à la nouveauté tant dans la consommation de biens culturels que dans les innovations technologiques. Dans la vaisselle de table, mais également dans la céramique liée à l'hygiène corporelle, il semble qu'on puisse trouver des critères de distinction entre les milieux parce que ce sont essentiellement dans ces deux catégories d'objets domestiques que sont introduites les produits les plus nouveaux, les plus innovants d'un point de vue technique (Ravoire, 2000). Quant à l'introduction de biens exotiques, on est loin, à Paris, des nombreuses variétés de céramiques venant d'Italie, d'Espagne, voire du Proche-Orient qui, depuis le XII ${ }^{\mathrm{e}}$ siècle et durant de nombreuses décennies, viennent enrichir le vaisselier des Marseillais! (Collectif, 1995 ; Abel, 2011). Paris, capitale du royaume, est peu alimenté par des produits exogènes. Les potiers parisiens crient d'ailleurs au scandale à la vente de poterie des «Flandres » (grès rhénan ou faïence de Delft ?). La consommation est marquée, à partir du $\mathrm{XVII}^{\mathrm{e}}$ siècle, par l'introduction et la généralisation, dans la plupart des milieux, mais en des quantités très variables (Ravoire, 2002a, 2007b), de faïences, produites à Nevers et vendues par les marchands potiers parisiens. Au début du siècle, cette vaisselle est sans nul doute réservée exclusivement aux milieux aisés. La vaisselle de table, mais également de toilette, retrouvée dans la fouille du pavillon du roi à Versailles en est une illustration. Dans la seconde moitié du siècle, elle est déjà beaucoup plus répandue. La porcelaine, depuis le Moyen Âge, constitue le mobilier céramique rare, précieux et recherché. Il est très peu fréquent d'en rencontrer avant le XVIII ${ }^{\mathrm{e}}$ siècle, mais cela arrive (dans un contexte de la fin du $\mathrm{XVI}^{\mathrm{e}}$ siècle et dans un autre de la première moitié du XVII ${ }^{\mathrm{e}}$ siècle de la Cour Napoléon du Louvre : Bresc-Bautier, 2011). La porcelaine chinoise est considérée, par les archéologues néerlandais, comme un très bon marqueur de haut statut social quand il provient d'occupations du XVII ${ }^{\mathrm{e}}$ siècle (Courtney, 2010, p. 324). En revanche, au XVIII ${ }^{\mathrm{e}}$ siècle, elle est considérée comme de moins haute valeur en raison de la grande diffusion de ces céramiques sur les marchés (grâce à la très puissance Compagnie hollandaise des Indes orientales). Pourtant, elle reste, à Paris, réservée à l'aristocratie ou à des milieux aisés. Les contextes ordinaires n'en livrent pas. Cela tient sans doute à une distribution différente de cette vaisselle en France. L'introduction, vers le milieu du XVIII ${ }^{\mathrm{e}}$ siècle, de nouveaux produits comme la porcelaine tendre ou dure européenne, la faïence fine anglaise (et, dans une mesure encore peu connue, française) sont autant de marqueurs pour des populations qui veulent se distinguer par la qualité de leur mode de consommation. Les estimations archéologiques rejoignent, pour 
le XVIII ${ }^{\mathrm{e}}$ siècle, celles figurant dans les sources historiques qui concernent désormais un large panel sociologique.

Comme le disait justement Jean-Marie Pesez : «Il n'est cependant pas tout à fait sûr que l'archéologie soit la voie royale pour approcher la culture matérielle. En revanche, il est certain que restituer la culture matérielle est le dessein privilégié de l'archéologie» (Pesez, 1997, p. 66). La manière d'aborder ces études sur le mobilier doit, comme le dit fort justement Myriam Arcangeli à propos de ces travaux sur la culture matérielle antillaise du XVIII ${ }^{\mathrm{e}}$ siècle, passer par une analyse des «processus de création de chaque type de ressources. S'il faut prendre en compte la taphonomie des sites et le contexte archéologique pour comprendre les dépôts culturels, il faut aussi évaluer les inventaires en fonction des conditions de création de l'acte» (Arcangeli, 2007). Nous devons nous servir du mobilier archéologique comme estimateurs de richesse avec les mêmes réserves que l'historien qui analysera les mobiliers inventoriés dans les actes notariés.

Il n'est donc pas possible de prendre la découverte dans un contexte archéologique d'un d'objet considéré comme de plus haute valeur marchande qu'une poterie ordinaire, comme suffisant pour prétendre que le contexte dont il est issu est socialement supérieur à d'autres qui en sont dépourvus. Par contre, l'observation systématique de phénomènes récurrents, dans une même aire géographique, dans un même contexte socioculturel, à une même période, de la présence de certaines catégories de mobilier peut avoir un sens social. La découverte d'un objet en faïence $a u \mathrm{XV}^{\mathrm{e}}$, $\mathrm{XVI}^{\mathrm{e}}$ ou $\mathrm{XVII}^{\mathrm{e}}$ siècle ne permet pas d'identifier nécessairement un milieu aisé, mais une grande quantité de cette vaisselle peut signaler, selon le type de mobilier, une apothicairerie si ce sont albarelles et pots canons, chevrettes qui sont retrouvés, si ce sont des plats, des assiettes, des coupes, un habitat aristocratique ou de la haute bourgeoisie, mais aussi une communauté monastique! (Ravoire, 2006b). C'est l'accumulation de plusieurs estimateurs qui permet de valider le caractère de marqueur social du mobilier céramique. Dans de rares cas seulement, la découverte de céramique à très haute valeur ajoutée, peut être suffisante. Enfin, la confrontation avec les autres sources archéologiques, en particulier celles qui se rapportent à la consommation alimentaire, est plus que nécessaire pour arriver véritablement à caractériser nos contextes. La comparaison avec d'autres mobiliers comme la verrerie ou le métal est également importante, surtout si elle se rapporte aux mêmes de domaines.
ABEL V., 1987 : «La Céramique commune à Marseille au XVII ${ }^{\mathrm{e}}$ siècle : l'exemple de dépotoirs domestiques du site de la Charité », Archéologie du Midi Médiéval, t. V, p. 153-165.

ARCHANGELI M., 2007, «Canaris et services à café : les céramiques des Antilles au XVIII ${ }^{\mathrm{e}}$ siècle", Actes du colloque Medieval Europe in Paris, Paris, septembre 2007. http://medieval-europe-paris-2007. univ-paris1.fr

AleXANDRE-Bidon D., 2005 : Une archéologie du goût. Céramique et consommation (Moyen Âge - Temps modernes), Paris, Picard, $301 \mathrm{p}$.

BARAY L., 2003 : Pratiques funéraires et sociétés de l'âge du Fer dans le Bassin parisien (dernier quart du viie s.-troisième quart du iie s. av. J.-C.), Gallia, (supplément 56), Paris, éd. du CNRS, 454 p.

Baray L., Brun P., A. TestartA. (DIR.), 2007 : Pratiques funéraires et sociétés. Nouvelles approches en archéologie et en anthropologie sociale, Dijon, Publication de l'université de Dijon (coll. Art, Archéologie et Patrimoine), p. 169-189.

BIMBENET-PRIVAT M., 2009: «Vaisselle utilitaire d'orfèvrerie au XVI ${ }^{\mathrm{e}}$ siècle», in Ravoire F. (DIR), Dietrich A. (DIR), p. 149-156.

Bimbenet-Privat M. (DIR.), 2011 : « Trésors enfouis de la Renaissance. Autour de Pouilly-sur-Meuse, catalogue de l'exposition Un exceptionnel ensemble d'orfèvrerie Renaissance. Le trésor de Pouillysur-Meuse, musée Lorrain à Nancy, éd. RMN Grand-palais, 127 p.

BOURQUIN L., 1989: « Les objets de la vie quotidienne dans la première moitié du $\mathrm{XVI}^{\mathrm{e}}$ siècle à travers cent inventaires après décès parisiens », Revue d'Histoire moderne et contemporaine, XXXVI, p. 465-475.

Bourquin L., HAMON P. (DIR.), 2011 : « Fortunes urbaines », Presses Universitaires de Rennes, $218 \mathrm{p}$.

Bresc-Bautier G. (DIR.) 2011 : Archéologie du Grand Louvre,

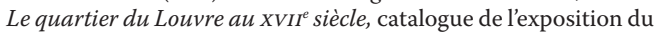
16 mars-31 décembre 2001, musée du Louvre.

Burnouf J., Laurioux B., Verhaeghe F., 2009, « Un colloque qui vient à point », in Ravoire F. (DIR.), Dietrich A. (DIR.), p. 1-8.

Chami J., 1963: «L'art céramique du Beauvaisis », Cahiers de la Céramique, du verre et des Arts du Feu, n ${ }^{\circ}$ 30, Sèvres.

Collectif, 1995 : Le vert et le Brun, De Kairouan à Avignon, céramiques du xe au xve siècle, catalogue de l'exposition du musée de Marseille, Musées de Marseille et RMN, 246 p.

Courtney P., 2010 : « Social theory and post-medieval archaeology: a historical perspective », in VerHAEgHE F., Exchanging Medieval Material Culture, p. 317-345.

Crepin-Leblond T., 1998 : Une orfèvrerie de terre, Bernard Palissy et la céramique de Saint-Porchaire, catalogue de l'exposition du musée national de la renaissance, château d'Ecouen, 1997-1998, 143 p.

Dietrich A., 2009: « Les petits métiers du bois et les arts de la table à la fin du Moyen âge », in Ravoire F. (DIR.), Dietrich A. (DIR.), p. 157-165.

Durey-Blary V. 1993 : Céramiques du xive siècle trouvées dans un dépotoir de l'abbaye de Maubuisson, Archéologie en Val-d'Oise, n ${ }^{\circ} 4$, Saint-Ouen-l'Aumône, Service départemental d'archéologie du Val-d'Oise, $144 \mathrm{p}$.

GARNOT B., 1995, La culture matérielle en France aux XVI ${ }^{e}, X V I I^{e}$ et XVIII ${ }^{e}$ siècles, Ophrys (collection Synthèse Histoire), 184 p.

GuAdAGnin R. 2007 : Fosses-Vallée de l'Ysieux, Mille ans de production céramique en Île-de-France, vol. 2, Caen, CRAHM, 735 p.

HodDER I., 1987: The Archaeology of contextual meanings, Cambridge University Press, $144 \mathrm{p}$.

Husi P., 2006: « La céramique peinte et glaçurée du Haut Moyen Âge dans l'Ouest de la France : nouvelles données pour d'anciennes interprétations, un bilan pour le Nord-Ouest de l'Europe », in HinCKer V., Husi P., La céramique du Haut Moyen Âge dans le Sud-Ouest de l'Europe $V^{e}$-X $X^{e}$ s., Acte du colloque de Caen, 18-20 mars 2004, Caen, NAE, p. 173-18o.

Husi P., 2009 : « De la table médiévale au vaisselier archéologique : l'exemple de la céramique du basin de la Loire moyenne », in Ravoire F. (DIR), DieTrich A. (DIR), p. 271-28o.

Journot F, Ravoire F., Horry A., Abel V. 2011, « Céramiques modernes », in Journot F. Belan G, (DIR.), Archéologie moderne et contemporaine en France, Paris, La Découverte, p. 93-104.

LAFARGE I, LeFÈVRE A. 2009 : « Éléments de la table et de la cuisine dans l'habitat de paysans aisés d'Île-de-France à travers les données archéologiques : l'exemple du site de la rue des Fossés du XII à la fin du XV siècle à Tremblay-en-France (Seine-Saint-Denis) », in Ravoire F. (DIR.), Dietrich A. (DIR.), p. 425-45o.

Lefèvre A., MAHÉ N., 2004 : « La céramique du haut Moyen Âge en Île-de-France à travers la fouille des habitats ruraux ( $\mathrm{VI}^{\mathrm{e}}-\mathrm{XI}^{\mathrm{e}}$ siècles). État de la question et perspectives de recherches ", Actes des journées d'étude d'Amiens Céramiques domestiques et terres cuites architecturales au Moyen Âge, 2001-2002-2003, Revue archéologique de Picardie, $\mathrm{n}^{\circ} 3 / 4$, p. 105-149.

Muel F., 2011 : « Trésor enfouis de la fin du Moyen Âge », in BimbenetPRIVAT M. (DIR.), Trésors enfouis de la Renaissance. Autour de Puilly-sur-Meuse, Paris, RMN, p. 106-113.

Nicourt J., 1986 : Céramiques médiévales parisiennes, classification et typologie, Paris, supplément aux publications de l'association Jeunesse Préhistorique et Géologique de France, 336 p.

Pardailhé-Galabrun A., 1988 : La naissance de l'intime. 3000 foyers parisiens, $X V I I^{e}-X I_{I I} I^{e}$ siècles, Paris, PUF, 524 p.

Pendery S., 2007: « La vie matérielle aux centres urbains des colonies anglaises ", Actes du colloque Medieval Europe in Paris, Paris septembre 2007. http://medieval-europe-paris-2007.univ-paris1.fr

Pesez J.-M., 1997 : L'archéologie, Mutations, missions, méthodes, Nathan Université (collection Histoire, 128), 128 p. 
Piponnier F., 1987 : « La céramique dans son contexte quotidien d'après les inventaires bourguignons ( $\mathrm{XIV}^{\mathrm{e}}-\mathrm{XV}^{\mathrm{e}}$ siècles) », in CHAPELOT J., Galinié H., Pilet-Lemière J. (ÉD.), Actes du colloque La céramique $\left(V^{e}-X I X^{e} s.\right)$ Fabrication-Commercialisation-Utilisation, $1^{\mathrm{er}}$ congrès International d'Archéologie Médiévale, Caen, p. 235-243.

Ravoire F. Monnet C., 1992 : « Les fosses à fond perdu, miroirs du quotidien et de l'exceptionnel », in MonNet C. (DIR.), Lévacuation des déchets en milieu urbain au bas Moyen Âge, l'exemple de la Cour Napoléon du Louvre, Louvain-La-Neuve, Université catholique de Louvain, p. 33-65.

RAvoire F., 1998a : " La vaisselle en terre cuite ", in VAN OsSEL P. (DIR.), Les Jardins du Carrousel (Paris). De la campagne à la ville : la formation d'un espace urbain, Paris, éd. de la Maison des sciences de l'homme (coll. Archéologie préventive, DAF, 73), p. 240-248.

RAVOIRE F., 1998b : « La céramique du XVI ${ }^{\mathrm{e}}$ et du début du XVII ${ }^{\mathrm{e}}$ siècle issue des fortifications ", in VAN OSSEL P. (DIR.), Les Jardins $d u$ Carrousel (Paris). De la campagne à la ville : la formation d'un espace urbain, Paris, éd. de la Maison des sciences de l'homme (coll. Archéologie préventive, DAF, 73), p. 172-185.

RAVOIRE F., 2000 : "Aperçu sur l'artisanat de la poterie de terre en Île-de-France entre le XIII ${ }^{\mathrm{e}}$ et le XVII ${ }^{\mathrm{e}}$ siècle ", in Utilis est lapis in structura, Mélanges offerts à Léon Pressouyre, Paris, éd. du CTHSp. p. 447-46o.

RAVOIRE F., 2002a : « Distribution et consommation de la majolique d'origine française, italienne et des Pays-Bas en France à la Renaissance ", Majolique et verre : de l'Italie à Anvers et au-delà. $L$ a diffusion de la technologie au XVI ${ }^{e}$ siècle et au début du XVII siècle, Actes du Colloque international d'Anvers, juin 1999, p. 347-370.

RAVOIRE F., 2002b : «Céramique importée et différenciation sociale : l'exemple de la vaisselle parisienne à la Renaissance (fin du XV $\mathrm{XV}^{\mathrm{e}} \mathrm{s} .-\mathrm{XVI} \mathrm{I}^{\mathrm{e}} \mathrm{s}$.) ", in Helmig G., Scholkmann B., Untermann M. (ÉD.), CentreRegion-Periphery: Medieval Europe Basel 2002, Actes du Colloque international d'archéologie médiévale et post-médiévale de Bâle, 10-15 septembre 2002, Bâle, Hertingen, Folio-Verlag., vol. 3, p. 364-373.

RAVOIRE F., 2006a : « Typologie raisonnée des céramiques de la fin du Moyen Âge et du début de l'époque moderne provenant du Beauvaisis, de Paris et d'ailleurs retrouvées sur les sites de consommation parisiens et franciliens ", Revue Archéologique de Picardie, $\mathrm{n}^{\circ}$ 3-4, p. 105-202.

RAVOIRE F., 2006b : «Approvisionnement céramique et mode d'alimentation dans les communautés religieuses sous l'Ancien régime. L'exemple de l'abbaye de Chelles (Seine-et-Marne) et du couvent des Feuillantines à Paris (VI $\mathrm{e}^{\mathrm{a}}$ arr.) ", in Clavel B. (DIR.), Production alimentaire et lieux de consommation dans les établissements religieux au Moyen Âge et à lépoque Moderne, Actes du colloque de Lille, 16-19 octobre 2003, CAHMER (Université de Picardie) et CREDHIR (Université catholique de Lille), Histoire médiévale et archéologie, $\mathrm{n}^{\circ}$ 19, p. 301-325.

RAVOIRE F., 2007a : «Évolution du confort matériel dans les maisons paysannes sous l'Ancien Régime en Île-de-France (XVI ${ }^{\mathrm{e}}$-XVIII ${ }^{\mathrm{e}} \mathrm{s}$.) l'apport des sources archéologiques ", in TROCHET J.-R. (DIR.), Actes du colloque Les maison paysannes en Europe occidentale, du la fin du Moyen Âge au XIX $X^{e}$ s., Paris 14-16 septembre 2006, Paris, Université Paris-Sorbonne, Institut de géographie, Presses Universitaires de Paris-Sorbonne.

RaVOIRE F., 2007b : « Mobilier céramique des XVII ${ }^{e}$ et XVIII ${ }^{e}$ s., approche sociale : l'apport des fouilles archéologiques d'île-de-France », Actes $d u$ Colloque international Medieval Europe in Paris, Paris, 3-8 septembre 2007. http://medieval-europe-paris-2007.univ-paris1.fr/ $\mathrm{MEP} 2007$

RAVOIRE F., 2008 : « Pour une archéologie du quotidien : objets et céramiques modernes ", in Archéopages, hors-série, p. 33-35.

RAVOIRE F., 2009: «Les productions céramiques d'Île-de-France à la fin du Moyen Âge (XIV ${ }^{\mathrm{e}}$-XVI ${ }^{\mathrm{e}}$ siècle), modalités et formes d'une évolution », in RAVOIRE F. (DIR), DieTRICH A. (DIR), p. 249-270.

RAVOIRE F. (DIR), DiETRICH A. (DIR), 2009 : La cuisine et la table dan la France de la fin du Moyen Âge. Contenus et contenants du XIV au XVI ${ }^{e}$ siècle, Actes du colloque de Sens, 8-10 janvier 2004, Caen, CRAHM, $450 \mathrm{p}$.

Ravoire F., 2011, "La céramique ", in Saint-Jean Vitus B. (Dir.), Bourgogne, Côte-d'Or, Dijon, Place de la République. Les dessous du tram : de la "rue des Eguletiers " à la porte Saint-Nicolas" (XIV $X V I^{e}$ s.), Dijon, Inrap, rapport dactylographié, p. 150-170.

RAVOIRE F., 2012: «Le mobilier céramique comme estimateur de richesse ", in HURARD S. (DIR.), La ferme du Colombier à Varennessur-Seine (Seine-et-Marne). Habitat rural et petites élites du XVI $I^{e}$ uu $X V I I I^{e}$ siècle : l'expression matérielle de l'ascension sociale d'une petite élite rurale, éd. du CNRS et Inrap (collection « Recherches archéologiques »).

Roche D., 1981 : Le peuple de Paris, Paris, éd. Aubier-Montaigne, 288 p.

VerhaEghe F, 1996 : «Aspects sociaux et économiques de la céramique très décorée : quelques réflexions ", in PITON D. (DIR.), La céramique très décorée dans l'Europe du Nord-Ouest ( $\mathrm{X}^{e}-\mathrm{XV} \mathrm{V}^{e}$ siècle), Actes du colloque de Douai, 1995, travaux du Groupe de recherches et d'études sur la céramique dans le Nord-Pas de Calais (Nord-Ouest Archéologie, 7), p. 233-247.

WARO-DESJARDINS F., 1992 : La vie quotidienne dans le Vexin au XVII siècle dans l'intimité d'une société rurale, éd. du Valhermeil-Société Historique de Pontoise, $542 \mathrm{p}$. 